\title{
28
}

\section{Performance evaluation of a JIT production system}

\author{
Mitsutoshi Kojima†, Katsuhisa Ohno† and Kenichi Nakashimał \\ †Department of Systems Engineering, \\ Nagoya Institute of Technology \\ Gokiso-cho, Showa-ku, Nagoya, 466, JAPAN, \\ Tel: $+81-52-735-5403$ and 5390 \\ Fax: $+81-52-735-5401$ and 5390 \\ E-mail:kojima@system.nitech.ac.jp, \\ ohno@system.nitech.ac.jp \\ $\ddagger$ Department of Industrial Management \\ Osaka Institute of Technology \\ 5-16-1, Omiya, Asahi-ku, Osaka, 535, JAPAN, \\ Tel: $+81-6-954-4788$ \\ Fax: +81-6-952-6197 \\ E-mail: nakasima@taylor.dim.oit.ac.jp
}

\begin{abstract}
In a Just-in-Time(JIT) production system, two kinds of Kanbans are used as tools to control the production and withdrawal quantities in each stage. The number of Kanbans used in stages decide the performance of the JIT production system. This paper analyzes it under stochastic demand and deterministic processing times. An algorithm is devised for computing stationary distributions of its production quantities and total backlogged demand. This algorithm can determine optimal numbers of two kinds of Kanbans. A numerical example is computed by this algorithm.
\end{abstract}

\section{Keywords}

Just-In-Time production system, Performance evaluation, Optimization 


\section{INTRODUCTION}

A Just-In-Time(JIT) production system is invented to chiefly aim at cost reduction by eliminating unnecessary elements in production (Monden, 1993). In a JIT production system, two kinds of Kanbans are used to control the production and withdrawal quantities in each stage. They are production-ordering Kanbans and withdrawal Kanbans. In particular, a withdrawal Kanban used for a vendor is called a supplier Kanban.

The numbers of Kanbans used in each stage decide the performance of the JIT production system. In the case where the demand is deterministic, Bitran and Chang(1987) deal with a deterministic multi-stage JIT production system. They formulate a problem of determining the number of production-ordering Kanbans in each stages into a nonlinear mixed integer programming problem. In the case where the demand is stochastic, Kimura and Terada(1981) show by simulation how fluctuations of the demand influence the production and inventory in the preceding stages in a multi-stage JIT production system. Deleersnyder et al.(1989) analyze the effect of the number of Kanbans, the machine reliability, the demand variability and the safety stock requirements on the performance of a JIT production system by numerical computation based on a discrete time Markov process. Wang and Wang(1990) deal with a JIT production system as a Markovian queue under the condition that both interarrival times of the demand and processing times are exponentially distributed. Karmarker and Kekre(1989) study a two-stage JIT production system by using queueing theory and show its numerical results. Mitra and Mitrani $(1990,1991)$ show that a Kanban discipline dominates a classical production discipline in terms of throughput. Kirkavak and Dinçer(1996) develop an exact performance evaluation model for JIT production system using discretetime Markov processes and propose a computationally feasible approximate decomposition technique. Tayur $(1992,1993)$ derives structural properties of a multi-stage JIT production system using a generalized semi-Markov process.

Under stochastic demand and deterministic processing times, Ohno et al.,(1995) discuss a JIT production system with the production-ordering and supplier Kanbans and derive a probability generating function(p.g.f.) of the stationary distribution of the backlogged demand. They, however, do not obtain any results on the production quantity except its expectation. Cohen and Boxma(1983) develop complex valued function techniques for the analysis of queueing systems which need for their modeling a two-dimensional state space. In this paper, we consider the JIT production system with two kinds of Kanbans under stochastic demand and deterministic processing times to derive a p.g.f. of the stationary distribution of its production quantities and total backlogged demand as a two-dimensional complex valued function. An algorithm for computing the stationary distributions is devised based on the p.g.f. This algorithm can determine optimal numbers of two kinds of Kanbans. Stationary distributions of the production quantities and total backlogged demand are shown in a numerical example. 


\section{A JIT PRODUCTION SYSTEM}

In a JIT production system, two kinds of Kanbans are mainly used: a productionordering Kanban and a withdrawal Kanban which are attached to containers of parts. The withdrawal Kanban specifies the kind and quantity of the parts which the subsequent stage should withdraw from the preceding stage, while the productionordering Kanban specifies the kind and quantity of parts which the preceding stage must produce. The number of production-ordering Kanbans corresponds to the maximum possible stock by the container of the parts produced in the stage. Similarly, the number of withdrawal Kanbans corresponds to the maximum possible stock by the container of the parts used for production in the stage. As the number of Kanbans increases, the stock of the parts also increases and the dead stock occurs. Conversely, as the number of Kanbans decreases, the stock of the parts also decreases and the shortage may occur. Therefore, they have influence on the performance of the JIT production system such as its inventory level, backlogged demand and production quantities.

We consider a JIT production system with supplier and production-ordering Kanbans (Ohno, et al., 1995) which is shown in Figure 1. In this system, the demand is stochastic and the processing time is deterministic. The following notation is used:

$L:$ the lead time of the delivery,

$M$ : the number of production-ordering Kanbans,

$N$ : the number of supplier Kanbans,

$C:$ the production capacity of the stage,

$D_{k}$ : the demand in period $k$,

$B_{k}$ : the backlogged demand at the beginning of period $k$,

$I_{k}$ : the inventory level of the part at the beginning of period $k$,

$J_{k}:$ the number of the production-ordering Kanban in the production-ordering Kanban post at the beginning of period $k$,

$P_{k}$ : the production quantity in period $k$,

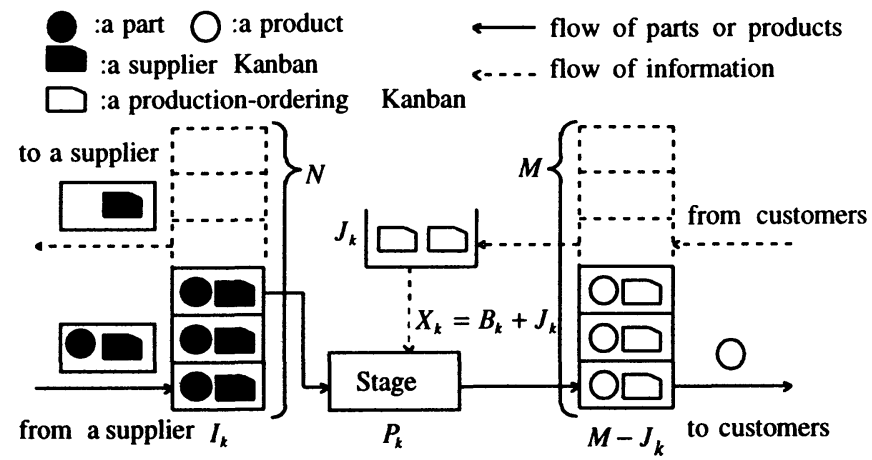

Figure $1 \mathrm{~A}$ JIT production system 
$X_{k}=B_{k}+J_{k}$ : the total backlogged demand at the beginning of period $k$.

The preceding stage is a supplier and the parts consumed in period $k(=1,2, \cdots)$ is ordered to the supplier at the beginning of period $k+1$ and they are delivered at the beginning of period $k+L+1$. It is assumed in the following of this paper that the demand of the product in each period is independent and identically distributed with mean $D$, the excess demand is backlogged and the container capacity is equal to one.

It holds (Ohno, et al., 1995) that for $k=1,2,3, \cdots$

$$
\begin{aligned}
& N=I_{k}+\sum_{i=k-L}^{k-1} P_{i}, \\
& I_{k+1}=I_{k}+P_{k-L}-P_{k}, \\
& P_{k}=\min \left(I_{k}, J_{k}, C\right), \\
& B_{k+1}=\left[B_{k}+J_{k}+D_{k}-P_{k}-M\right]^{+}, \\
& J_{k+1}=\min \left(M, B_{k}+J_{k}+D_{k}-P_{k}\right), \\
& X_{k+1}=X_{k}+D_{k}-P_{k},
\end{aligned}
$$

where $P_{0}, P_{-1}, \cdots, P_{-L+1}$ are given and $[x]^{+}=\max (0, x)$.

\section{THE PROBABILITY GENERATING FUNCTION OF THE STATIONARY DISTRIBUTION}

We derive a probability generating function of the stationary distribution of backlogged demand and production quantities in the JIT production system.

It follows from (3) and (6) that $X_{k}$ and $P_{k}, k=1,2,3, \ldots$ in the case of $N>(L+$ 1) $M^{\prime}$ ' have the same values as those in the case of $N=(L+1) M^{\prime}$, where $M^{\prime}=$ $\min (M, C)$. Therefore we can restrict without loss of generality to the case where $N^{2}$ $(L+1) M^{\prime}$. Define integers $m$ and $v$ by $N=m M^{\prime}-v, 1^{2} m^{2} L+1,0^{2} v^{2} M^{\prime}-1$.

It is shown in Ohno, et al., (1995) that for $n=1,2,3, \cdots$

$$
\begin{gathered}
Y_{n}=X_{n(L+1)+1}=\max \left\{D_{n(L+1)}, D_{n(L+1)}+D_{n(L+1)-1}-M^{\prime}, \ldots, \sum_{i=L-m+2}^{L+1} D_{(n-1)(L+1)+i}-(m-1) M,\right. \\
\left.Y_{n-1}+\sum_{i=1}^{L+1} D_{(n-1)(L+1)+i}-N\right\} .
\end{gathered}
$$

Define random variables $Y_{n-1}(k)$ and $I_{n-1}(k), n=1,2,3, \cdots, 0^{2} k^{2} m$, by

$$
\begin{aligned}
& Y_{n-1}(0)=Y_{n-1}, Y_{n-1}(1)=\left[Y_{n-1}(0)+\sum_{i=1}^{L-m+1} D_{(n-1)(L+1)+i}-M^{\top}+v\right]^{+}, \\
& Y_{n-1}(k)=\left[Y_{n-1}(k-1)+D_{n(L+1)+k-m-1}-M^{\prime}\right]^{+}, \\
& I_{n-1}(0)=P_{(n-1)(L+1)+1}, I_{n-1}(1)=\left[M-v-Y_{n-1}(0)-\sum_{i=1}^{L-m+1} D_{(n-1)(L+1)+i}\right]^{+}+I_{n-1}(0)
\end{aligned}
$$

and

$$
I_{n-1}(k)=\min \left\{\left[M^{\prime}-Y_{n-1}(k-1)-D_{n(L+1)+k-m-1}\right]^{+}+I_{n-1}(k-1), M\right\} .
$$


Since $I_{n(L+1)+1}=N-\sum_{i=2}^{L+1} P_{(n-1)(L+1)+i}$

$=N-\left(\sum_{i=1}^{L+1} D_{(n-1)(L+1)+i}+X_{(n-1)(L+1)+1}-X_{n(L+1)+1}\right)+P_{(n-1)(L+1)+1}$

and

$(m-k) M+X_{n(L+1)+1}-\sum_{i=L-m+k+1}^{L+1} D_{(n-1)(L+1)+i}-Y_{n-1}(k) \geq 0,1 \leq k \leq m$,

(7) through (9) imply that

$Y_{n}=Y_{n-1}(m)+D_{n(L+1)}$,

and

$P_{n(L+1)+1}=\min \left\{Y_{n-1}(m)+D_{n(L+1)}, I_{n-1}(m)\right\}$.

The JIT production system is called stable, if $Y_{n-1}(0)=Y_{n-1}$ and $I_{n-1}(0)=P_{(n-1)(L+1)+1}$ converge in distribution to $Y(0)=X_{\infty}$ and $I(0)=P_{\infty}$, respectively, where $\left(X_{\infty}, P_{\infty}\right)$ is distributed with the stationary distribution. The following condition is derived by Ohno, et al., (1995):

If $\operatorname{Pr}\left\{D_{k}-D\right\}>0$, then the JIT production system is stable if and only if $\min \{C, M, N /(L+1)\}>D$.

Denote the p.g.f. of a random vector $\left(X_{\infty}, P_{\infty}\right)$ by $X(z, w)=E\left[z^{Y_{\infty}} w^{P_{\infty}}\right]$. Then it can be shown from (10) and (11) that $X(z, w)=$

$$
\begin{aligned}
& \left\{z^{M^{\prime}-v} D(z)^{m} \sum_{x=0}^{M^{\prime}-\nu-1} \sum_{i=0}^{x} \sum_{d=0}^{M^{\prime}-v-1-x}\left(w^{i+M^{\prime}-v-x-d}-z^{x+d-M^{\prime}+v} w^{i}\right)\right. \\
& \times \operatorname{Pr}\{Y(0)=x, I(0)=i\} \operatorname{Pr}\left\{\sum_{k=1}^{L-m+1} D_{k}=d\right\}
\end{aligned}
$$

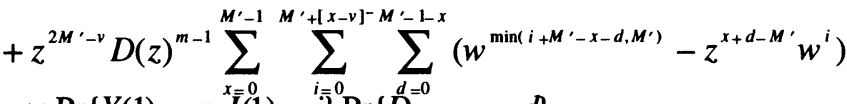

$$
\begin{aligned}
& \times \operatorname{Pr}\left\{Y(1)=x, \stackrel{x=0}{\bar{I}(1)}=\stackrel{i=0}{i=} \operatorname{Pr}\left\{D_{L_{-m+2}}^{d=0}=d\right\}\right. \\
& \begin{array}{l}
+\sum_{k=2}^{m-1} z^{(k+1) M^{\prime}-v} D(z)^{m-k} \sum_{x=0}^{M^{\prime}-1} \sum_{i=0}^{M^{\prime}} \sum_{d=0}^{M^{\prime}-L^{-x}}\left(w^{\min \left(i+M^{\prime}-x-d, M^{\prime}\right)}-z^{x+d-M^{\prime}} w^{i}\right) \\
\quad \times \operatorname{Pr}\{Y(k)=x, I(k)=i\} \operatorname{Pr}\left\{D_{L+1-m+k}=d\right\}
\end{array} \\
& \left.+\sum_{x=0}^{M^{\prime}-1} \sum_{i=x+1}^{M^{\prime}} \sum_{d=0}^{M^{\prime}-x-1} z^{x+d}\left(w^{x+d}-w^{i}\right) \times \operatorname{Pr}\{Y(m)=x, I(m)=i\} \operatorname{Pr}\left\{D_{L+1}=d\right\}\right\} \\
& /\left\{z^{N}-D(z)^{L+1}\right\}
\end{aligned}
$$

where

$$
\begin{aligned}
& Y(0)=X_{\infty}, Y(1)=\left[Y(0)+\sum_{i=1}^{L-m+1} D_{i}-M+v\right]^{+}, \\
& Y(k)=\left[Y(k-1)+D_{L+k-m}-M\right]^{+}, 2 \leq k \leq m, \\
& I(0)=P_{\infty}, I(1)=\left[M-v-Y(0)-\sum_{i=1}^{L-m+1} D_{i}\right]^{+}+I(0), \\
& I(k)=\min \left\{\left[M-Y(k-1)-D_{L+k-m}\right]^{+}+I(k-1), M\right\} 2 \leq k \leq m,
\end{aligned}
$$


$D(z)=E\left[z^{D_{k}}\right]$ for $k=1,2,3, \cdots,|z|^{2} 1,|w|^{2} 1,[x]^{-}=\min (0, x)$ and if $m=2(m=1)$, the third (and the second) term of the numerator in (13) vanishes.

\section{AN ALGORITHM FOR COMPUTING THE PERFORMANCE EVALUATION}

We devise an algorithm for computing the stationary distributions based on the p.g.f. (13).

Define functions $\alpha(z, w)$ and $\beta(z)$ by the numerator and denominator of the p.g.f. $X(z, w)$, respectively. The p.g.f. $X(z, w)$ has $N \times\left(M^{\prime}+1\right)$ unknown values:

$\operatorname{Pr}\{Y(0)=x, I(0)=i\} 0 \leq x \leq M-v-1,0 \leq i \leq x$, $\operatorname{Pr}\{Y(1)=x, I(1)=i\} \quad 0 \leq x \leq M^{\prime}-1,0 \leq i \leq M^{\prime}+[x-v]^{-}$,

$\operatorname{Pr}\{Y(k)=x, I(k)=i\} \quad 0 \leq x \leq M^{\prime}-1,0 \leq i \leq M, 2 \leq k \leq m-1$, and

The probability generating functions $E\left[z^{X_{\infty}}\right]$ and $E\left[w^{R_{\infty}}\right]$ are given by L'Hospital's theorem as follows:

$E\left[z^{X_{\infty}}\right]=X(z, 1)$ and $E\left[w^{P_{\infty}}\right]=\lim _{z \rightarrow 1} X(z, w)=\alpha^{\prime}(1, w) / \beta^{\prime}(1)$.

where $\alpha^{\prime}(z, w)$ and $\beta^{\prime}(z)$ denote the first derivatives of $\alpha$ and $\beta$ with respect to $z$, respectively. From Rouche's theorem and the continuity of $E\left[w^{p_{\infty}}\right]\left(|w|^{2} 1\right), \alpha$ $(z, w)$ satisfies the following identities that $\alpha\left(z_{n}, w\right)=0,1 \leq n \leq N,|w| \leq 1$, where $z_{n},\left|z_{n}\right|<1$ for $1 \leq n \leq N-1$ and $z_{N}=1$ are roots of the equation $\beta(z)=0$.

Define $\alpha_{k}\left(z_{n}\right), 0 \leq k \leq M^{\prime}$ as the coefficient of $w^{k}$ in $\alpha\left(z_{n}, w\right)$. Then we can determine the unknown values (15) by solving the following system of $N \times$ $\left(M^{\prime}+1\right)+1$ linear equations :

$\alpha^{\prime}(1,1)=\beta^{\prime}(1)$ and $\alpha_{k}\left(z_{n}\right)=0,0 \leq k \leq M, 1 \leq n \leq N$,

where one of the equations $\alpha_{k}\left(z_{n}\right)=0$ is redundant.

Moreover, the expectation $E\left[X_{\infty}\right]$ is also obtained by

$$
E\left[X_{\infty}\right]=\lim _{z \rightarrow 1} \frac{d}{d z} X(z, 1)=\left\{\alpha^{\prime \prime}(1,1)-\beta^{\prime \prime}(1)\right\} / 2 \beta^{\prime}(1)
$$

where $\alpha "(z, w)$ and $\beta$ " $(z)$ denote the second derivatives of $\alpha$ and $\beta$ with respect to $z$, respectively. It follows from (6) that

$E\left[P_{\infty}\right]=D$

and

$$
\operatorname{Pr}\left\{P_{\infty}=k\right\}=\alpha_{k}(1) / \beta(1), 0 \leq k \leq M^{\prime},
$$

where $\alpha_{k}(1)$ is the coefficient of $w^{k}$ in $\alpha(1, w)$.

The basic relation among $B_{k}, J_{k}$ and $X_{k}$ implies that $E\left[J_{\infty}\right]=\sum_{i=0}^{M-1} i P\left\{X_{\infty}=i\right\}+M\left(1-\sum_{i=0}^{M-1} \operatorname{Pr}\left\{X_{\infty}=i\right\}\right), \quad E\left[B_{\infty}\right]=E\left[X_{\infty}\right]-E\left[J_{\infty}\right]$ 
and $\operatorname{Pr}\left\{B_{\infty}>0\right\}=1-\sum_{i=0}^{M} P\left\{X_{\infty}=i\right\}$,

where the values $\operatorname{Pr}\left\{X_{\infty}=i\right\}$, that is, $\operatorname{Pr}\{Y(0)=i\}, 0^{2} i^{2} M$, can be determined by (16).

Moreover, we obtain from (1) and (19) that

$E\left[I_{\infty}\right]=N-L D$.

Therefore, an algorithm for computing the performance of the JIT production system is as follows:

Step1. Determine roots $z_{n},\left|z_{n}\right|<1$ for $1^{2} n^{2} N-1$ of the equation $z^{N}-D(z)^{L+1}=0$ by an appropriate method such as Newton's method.

Step2. Determine $N \times\left(M^{\prime}+1\right)$ unknown values:

$\operatorname{Pr}\{Y(0)=x, I(0)=i\} \quad 0 \leq x \leq M-v-1,0 \leq i \leq x$,

$\operatorname{Pr}\{Y(1)=x, I(1)=i\} \quad 0 \leq x \leq M^{\prime}-1,0 \leq i \leq M^{\prime}+[x-v]^{-}$,

$\operatorname{Pr}\{Y(k)=x, I(k)=i\} \quad 0 \leq x \leq M^{\prime}-1,0 \leq i \leq M, 2 \leq k \leq m-1$,

and

$\operatorname{Pr}\{Y(m)=x, I(m)=i\} \quad 0 \leq x \leq M-1, x+1 \leq i \leq M$,

by solving (17).

Step3. Compute $E\left[X_{\infty}\right], E\left[P_{\infty}\right], E\left[J_{\infty}\right], E\left[B_{\infty}\right], E\left[I_{\infty}\right], \operatorname{Pr}\left\{B_{\infty}>0\right\}, \operatorname{Pr}\left\{P_{\infty}=i\right\}$ $0^{2} i^{2} M^{\prime}$ by (14), (18) through (22).

\section{OPTIMIZATION OF THE NUMBERS OF KANBANS}

The JIT production system adapts to variable demands at a small cost by production smoothing (Monden, 1993). Through production smoothing, the stages can reduce idle time or overtime costs of workers or machines. We call these costs related to production quantities "production fluctuation costs" and include them in a cost function of the JIT production system.

Suppose that the ordered quantities, $P_{0}, \cdots, P_{-L}$ are given. Then, a standard cost function over $\mathrm{K}$ periods is as follows:

$$
\begin{aligned}
A(M, N, K)= & E\left[\sum _ { k = 1 } ^ { K } \left\{A_{l}\left(I_{k}-P_{k} / 2\right)+B_{I}\left(M-J_{k}\right)+A_{B} B_{k}\right.\right. \\
& +A_{o} P_{k-1}+A_{W} P_{k-L-1}+\sum_{i=0}^{M} A_{P}(i) \operatorname{Pr}\left\{P_{k}=i\right\} \\
& \left.\left.+C_{B} I\left\{B_{k}>0\right\}+C_{o w}(M, N)\right\}+A_{s}\left(I_{K}-P_{K}\right)+\sum_{i=1}^{L} A_{E}(i) P_{K-i}\right],
\end{aligned}
$$

where $I\{H\}$ is the indicator function of event $H$, that is, $I\{H\}=1$ if $H$ occurs; $=0$, otherwise. In addition,

$A_{I}$ : the inventory cost of one part per period,

$B_{I}$ : the inventory cost of one product per period,

$A_{B}$ : the backlogged cost of one product per period, 
$A_{o}:$ the ordering cost of one part,

$A_{w}$ : the withdrawing cost of one part,

$A_{P}(i)$ : the production fluctuation cost per period when the production quantity is $i$.

$C_{B}$ : the backlogged cost per once,

$A_{S}$ : the salvage cost of one part at the end of period $K$,

$A_{E}(i)$ : the salvage cost of one part elapsed $i$ periods after the ordering at the end of period $K$,

and

$C_{O W}(M, N)$ : the fixed cost per period of storage space, ordering and withdrawing when the numbers of Kanbans are $M$ and $N$.

We consider the average cost per periods over an infinite planning horizon in this paper. The average costs per period, $\mathrm{A}(M, N)$ is defined by

$A(M, N)=\limsup _{K \rightarrow \infty} A(M, N, K) / K$.

Under the stability condition (12), distributions of $B_{k}, I_{k}$, and $J_{k}$ also converge to their own stationary distributions, as $k$ tends to infinity, and denote by $B_{\infty}, I_{\infty}$, and $J_{\infty}$ random variables with the stationary distributions. Then it follows from (23) and (24) that

$$
\begin{aligned}
A(M, N) & =A_{I}\left(E\left[I_{\infty}\right]-E\left[P_{\infty}\right] / 2\right)+B_{I}\left(M-E\left[J_{\infty}\right]\right)+A_{B} E\left[B_{\infty}\right] \\
+ & \left(A_{o}+A_{W}\right) E\left[P_{\infty}\right]+\sum_{i=0}^{M} A_{P}(i) \operatorname{Pr}\left\{P_{\infty}=i\right\}+C_{B} \operatorname{Pr}\left\{B_{\infty}>0\right\}+C_{o w}(M, N)(1)
\end{aligned}
$$

Consider the case where $N>(L+1) M$ '. Since by (3), the production quantity in one period cannot exceed $M^{\prime}$, the $\left(N-(L+1) M^{\prime}\right)$ parts are always stored as the dead stock. That is, for $N>(L+1) M^{\prime}, A(M, N)=A\left(M,(L+1) M^{\prime}\right)+A_{l}\left(N-(L+1) M^{\prime}\right)$. Consequently, in the following of this paper, it suffices to consider only the case where $N^{2}(L+1) M$ '.

Thus, if we obtain the stationary distributions and expectations of random variables in (25) by the algorithm devised in Section 4, we can calculate the value of (25) and can determine optimal numbers of Kanbans, $M^{*}$ and $N^{*}$ that minimize $A(N, M)$.

\section{NUMERICAL EXAMPLE}

The algorithm devised in Section 4 is applied to the JIT production system with the average demand $D=7$, the lead time $L=4$ and the production capacity $C=10$. The stable condition (12) implies that $M>7$ and $N>35$. The distribution of the demand $D_{k}, k=1,2,3, \cdots$ is a shifted binomial distribution: 
$\operatorname{Pr}\left\{D_{k}=D-\frac{1}{2} n+j\right\}=\left(\begin{array}{l}n \\ j\end{array}\right)\left(\frac{1}{2}\right)^{n}, 0 \leq j \leq n$

where $n$ is an even number less than or equal to $2 D$. The expectation is $D$ and The variance is $n / 4$. For example, in the case where $n=8, \sigma^{2}$ is $n / 4=2.0$.

Figure 2 shows the distributions of the total backlogged demand as functions of $N$ with $M=10$. Clearly, decreasing the number of the supplier Kanbans increases

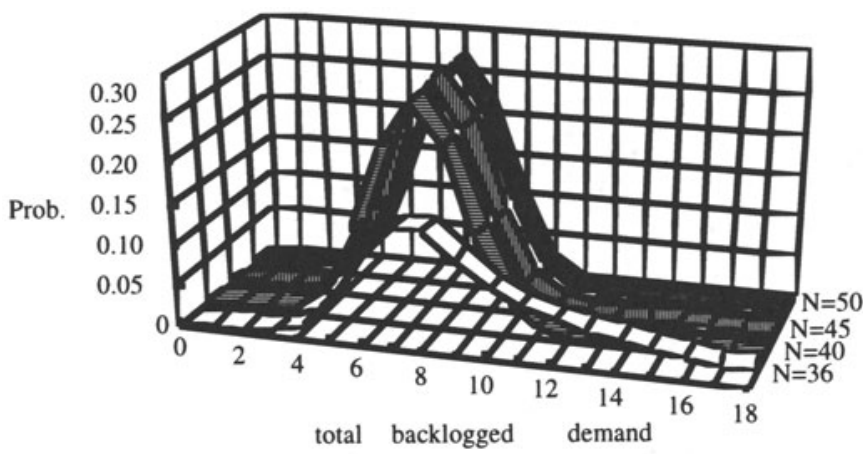

Figure 2 Probability distributions of the total backlogged demand for $M=10$

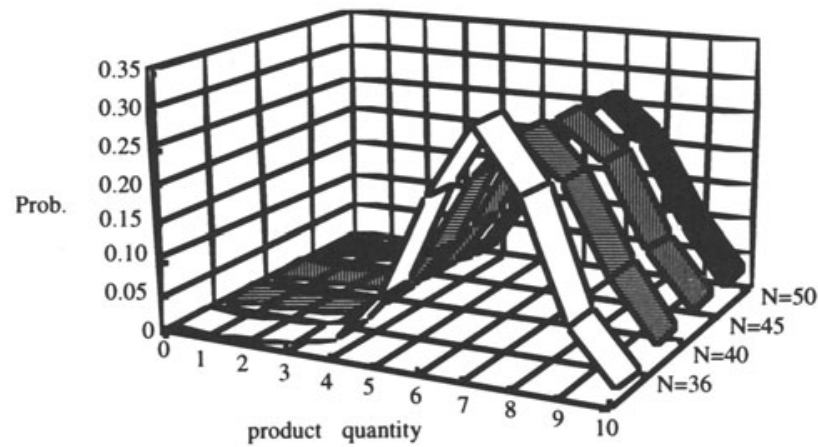

Figure 3 Probability distributions of the production quantities for $M=10$

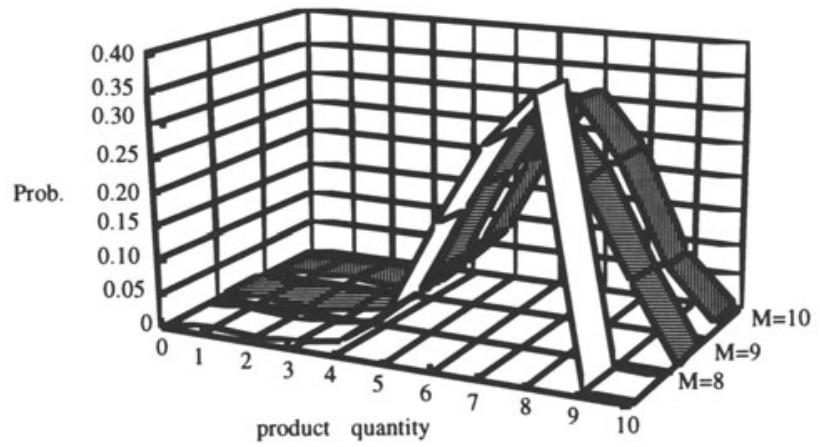

Figure 4 Probability distributions of the production quantities for $N=36$ 
the total backlogged demand. The expectations in the case where $N=36,40,45$ and 50 are $10.37,7.08,7.00$ and 7.00 , respectively.

Figure 3 shows the distributions of the production quantities as functions of $N$ in the same case as in Figure 2. The variance of the product quantities increases, as the number of supplier Kanbans increases. The variances in the case where $N=36$, 40,45 and 50 are $1.51,1.91,1,97$ and 1.97, respectively. Similar results are shown in Figure 4 in the case where $N=36$ and $M=8,9$ and 10.

The cost parameters in (25) are set as follows:

$A_{I}=1, B_{I}=10, A_{B}=0, A_{0}+A_{W}=1, C_{B}=100$,

$C_{\text {ow }}(M, N)=0$ and $A_{P}(i)=\left\{\begin{array}{cc}50(i-8) & 9 \leq i \leq 10 \\ 0 & 0 \leq i \leq 8\end{array}\right.$.

Figure 5 shows the behavior of costs in (25) as functions of $N$ with $M=10$. The inventory and ordering cost and the production fluctuation cost increase but the backlogged cost decreases, as $N$ increases.

Figure 6 shows the behavior of minimum average costs $A\left(M, N^{*}\right)$ as functions

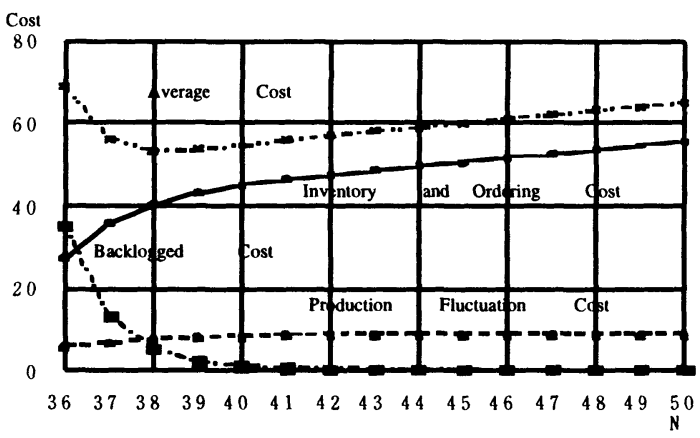

Figure 5 Behavior of costs for $M=10$

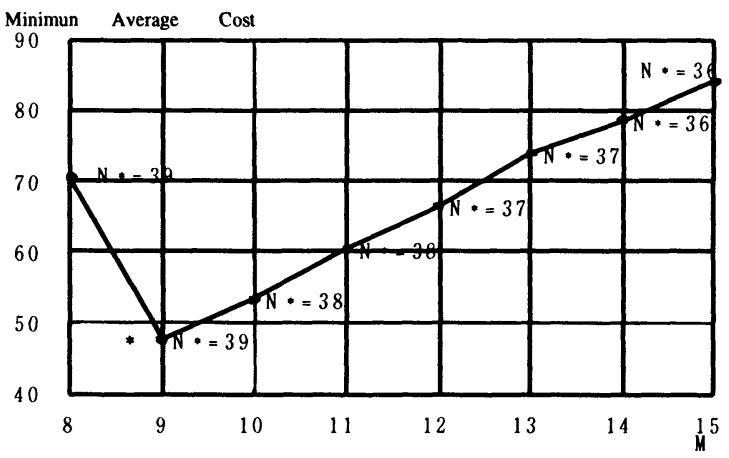

Figure 6 Behavior of $A\left(M, N^{*}\right)$ 
of $M$. The minimum average costs is attained at $M$ indicated by “*” in Figure 6 . This figure shows that the optimal numbers of production-ordering Kanbans and supplier Kanbans are $M^{*}=9$ and $N^{*}=39$, respectively.

\section{CONCLUSION}

In this paper, we deal with the JIT production system where the productionordering Kanbans and withdrawal Kanbans are used. Under the stochastic demand and deterministic processing time, the probability generating function of the stationary distribution of the total backlogged demand and the production quantities is derived as the two-dimensional complex valued function. The algorithm for computing the performance of the JIT production system is devised based on the p.g.f. Moreover, we consider the time average cost function with the production fluctuation cost and optimize numbers of two kinds of Kanbans. Numerical results illustrate the performance of the JIT production system and optimal numbers of two kinds of Kanbans are determined by using the devised algorithm.

\section{REFERENCES}

Bitran,G.R. and Chang,B. (1987), 'A mathematical programming approach to a deterministic kanban system', Management Science, 33, pp.427-441.

Cohen,J.W. and Boxma,O.J. (1983), Boundary Value Problems in Queueing System Analysis, North-Holland Publ. Co., Amsterdam.

Deleersnyder,J.L., Hodgson,T.J.,Muller(-Malek),H., and O'Grady,P.J. (1989), 'Kanban controlled pull systems: an analytic approach', Management Science, 35, pp.1079-1091.

Karmarker,U.S. and Kekre,S. (1989), 'Batching policy in kanban systems', Journal of Manufacturing Systems, 8, pp.317-328.

Kimura,O. and Terada,H. (1981), 'Design and analysis of pull system, a method of multi-process production control', International Journal of Production Research, 19, pp.241-253.

Kirkavak,N and Dinçer,C. (1996), 'Performance evaluation models for single-item periodic pull production systems', Journal of the Operational Research Society, 47, pp.239-250

Mitra,D. and Mitrani,I. (1990), 'Analysis of a kanban discipline for cell coordination in production lines. I', Management Science, 36, pp.1548-1566.

Mitra,D. and Mitrani,I. (1991), 'Analysis of a kanban discipline for cell coordination in production lines. II', Operations Research, 39, pp.807-823.

Monden,Y. (1993), Toyota Production System, Second Edition, Norcross,GA: Industrial Engineering and Management Press. 
Ohno,K., Nakashima,K and Kojima,M. (1995), 'Optimal numbers of two kinds of Kanbans in a JIT production system', International Journal of Production Research, 33, pp.1387-1401.

Tayur,S.R. (1992), 'Properties of serial kanban systems', Queueing Systems, 12, pp.297-318.

Tayur,S.R. (1993), 'Structural properties and a heuristic for kanban-controlled serial lines', Management Science, 39, pp.1347-1368.

Wang,H. and Wang,H.-P. (1990), 'Determining the number of kanbans: a step toward non-stock-production', International Journal of Production Research, 22, pp.2101-2115.

\section{BIOGRAPHY}

Mitsutoshi Kojima received B.S. and M.S. degrees in engineering from Nagoya Institute of Technology, Nagoya, Japan, in 1989 and 1991, respectively. From 1991 to 1994, he was a Computer Engineer with Fujitsu Limited, Numazu, Japan. Since 1994, he is a Research Associate with the Department of Systems Engineering, Nagoya Institute of Technology. His research interests include application of queueing theory to production systems and design and analysis of next generation production systems.

Katsuhisa Ohno received the B.S., M.S. and Ph.D. degrees in operations research from Kyoto University in 1964, 1966 and 1973, respectively. From 1966 to 1967, he was an Industrial Engineer with Toyota Motor Company and in 1967 he was a Research Assistant with Osaka University. From 1968 to 1984, he was a Research Assistant and then an Associate Professor with Kyoto University. From 1984 to 1986, he was a Professor with Konan University. Since 1986 he has been a Professor with Nagoya Institute of Technology. His research interests include queueing networks, dynamic programming, Markov decision processes, production management, Just-In-Time production systems and next generation production systems.

Prof. Ohno was a member of the Editorial Advisory Board of Transportation Research and an Associate Editor of J. Operations Research Soc. Japan. He is now Associate Editors of Belgian J. Oper. Res. Stat. Comp. Sci. and of Int. J. Engineering Design and Automation.

Kenichi Nakashima received B.S., M.S. and Ph.D. degrees in engineering from Nagoya Institute of Technology, Nagoya, Japan, in 1990, 1992 and 1995, respectively. From 1995 to 1996, he is a Research Associate with the Department of Industrial Management, Osaka Institute of Technology. Since 1996, he has been an Assistant Professor. His research interests include dynamic programming, Markov decision processes, production systems and management information systems. 